\author{
E. A. Mikhnevich, ${ }^{\mathrm{a}^{*}}$ A. P. Safronov, ${ }^{\mathrm{ab}}$ \\ I. V. Beketov, ${ }^{\text {ab }}$ A. I. Medvedev ${ }^{\text {ab }}$ \\ ${ }^{a}$ Ural Federal University, \\ 620002, 19 Mira St., Ekaterinburg, Russia \\ ${ }^{b}$ Institute of Electrophysics UB RAS, \\ 620016, 106 Amundsen St., Ekaterinburg, Russia \\ *email:emikhnevich93@gmail.com
}

\title{
Carbon coated Nickel Nanoparticles in Polyacrylamide Ferrogels: Interaction with Polymeric Network and Impact on Swelling
}

Polyacrylamide ferrogels with embedded magnetic nanoparticles of metallic nickel (Ni) and nanoparticles of nickel coated with a carbon shell (Ni@C) were synthesized by radical polymerization in water. The effect of the carbon shell on the interaction of $\mathrm{Ni}$ and $\mathrm{Ni@C} \mathrm{nanoparticles} \mathrm{with} \mathrm{polyacrylamide} \mathrm{matrix} \mathrm{and}$ on swelling ratio of the ferrogels has been studied. The deposition of carbon on the surface of $\mathrm{Ni}$ nanoparticles worsens their interaction with polyacrylamide but at the same time elevates the water uptake by ferrogels.

Keywords: nanoparticles; nickel; composites; ferrogels; polyacrylamide; carbon coatings.

Received: 26.08.2020. Accepted: 06.10.2020. Published: 07.10.2020.

(C) E. A. Mikhnevich, A.P. Safronov, I. V. Beketov, A. I. Medvedev, 2020

\section{Introduction}

A hydrogel is a three-dimensional polymeric network swollen in water. The internal structure of a hydrogel includes flexible polymeric sub-chains, which are crosslinked in a certain number of points. Due to the internal cross-linking the polymeric network of a hydrogel might be considered as a combined huge molecule. From the thermodynamic point of view hydrogel is a solution of this molecule in water. Despite the fact that it contains large amount of water, hydrogel is not a fluid but an elastic material maintaining its shape. Due to their unique properties such as mechanical elasticity, softness, and biocompatibility, hydrogels have been applied in a variety of fields, including smart devices that respond to stimuli and soft actuators in biomedicine and agriculture $[1,2]$.

Hydrogels are often functionalized by incorporating various physically, chemically, and biologically active moieties, which endows hydrogels with new functions, such as response to specific external stimuli and increased mechanical stability [3]. Stimulus-responsive hydrogels can markedly change their physical and/or chemical properties when exposed to external triggers $(\mathrm{pH}$, temperature, light, magnetic or electric field) [4]. For instance, the inclusion of magnetic nanoparticles (MNPs) in such a polymer network gives magnetic hydrogels (ferrogels) that react to an external magnetic field [5]. Ferro- 
gels are usually obtained by crosslinking hydrophilic monomers in a stabilized aqueous dispersion of magnetic nanoparticles (ferrofluid), which, in turn, provides good dispersion of nanoparticles in a polymer matrix. One of the main advantages of ferrogels over traditional stimulus-responsive polymers is that they can be remotely activated by a non-contact force (magnetic field). This unique property makes ferrogels prospective advanced material in various fields such as drug delivery $[6,7]$, soft robotics [8], tissue reconstruction $[9,10]$ and environmental engineering $[11,12]$.

Huang et al. [9] reported about a hydrogel formed by gelatin and $\beta$-cyclodextrin with embedded magnetic $\mathrm{Fe}_{3} \mathrm{O}_{4}$ nanoparticles for pulsed electromagnetic field therapy. Chondrogenesis of mesenchymal stem cells grown on a magnetic hydrogel was enhanced by a magnetic field. Czichy et al. [13] investigated the mechanical properties of alginate-methylcellulose hydrogels containing magnetite nanoparticles for the use in the additive manufacturing of implants. The study was an introduction to further research on the effect of an external magnetic field on the mechanical stability of composites. The most extensive study on ferrogels was carried out by Zrinyi et al. [14-16] on magnetic silicone elastomers filled with carbonyl iron or micronsized magnetite. The modulus of elasticity of these magnetoelastic gels was studied in both uniform and inhomogeneous magnetic fields. The influence of the content of magnetic particles and their distribution at various combinations of magnetic field orientation and deformation on the modulus has been systematically studied. Meanwhile, studies of synthetic water-based ferrogels are very limited.

In the literature most of the studies address ferrogels based on polyacrylamide
(PAAm) chemically cross-linked polymeric network. Thus, the series of works were performed by the group of Galicia et al [17-19] on ferrogels with embedded maghemite nanoparticles. Maghemite nanoparticles were synthesized using ferrofluid obtained by co-precipitation of iron oxides from $\mathrm{Fe}^{2+} / \mathrm{Fe}^{3+}$ mixed solutions. Ferrogels had weakly cross-linked polymeric network with monomer-to-cross-linker ratio 1000 and more and contained up to $7 \%$ (vol.) of nanoparticles. The structure of ferrogels in the swollen state and the mechanical properties were studied. It was shown that the Young modulus of ferrogels enlarged from 4 to $16 \mathrm{kPa}$ with the increase in nanoparticles content.

In our previous works [20-22] we have studied PAAm ferrogels with embedded iron and nickel nanoparticles, which were synthesized by the high-power pulsed physical dispersion by the electrical explosion of metal wire (EEW) [23]. The magnetostriction in the uniform magnetic field and the compression modulus of ferrogels were characterized. It was shown that ferrogels with iron nanoparticles contract by approximately $9 \%(\mathrm{vol})$ in the uniform magnetic field $420 \mathrm{mT}$ applied for 4 hours. Shear modulus of ferrogels with embedded iron nanoparticles increased from 0.5 to 2.5 $\mathrm{kPa}$ with the elevation of nanoparticles content up to $4 \%$ (vol.) [21]. In PAAm ferrogels with nickel nanoparticles it was shown [22] that the elastic modulus of ferrogels linearly depended on the content of the embedded nickel nanoparticles. The applied magnetic field 270 Oe in the parallel direction to the compression increased the elastic modulus by $10 \%$ while the application of the magnetic field in the transverse direction decreased the modulus.

Although ferrogels with embedded metal nanoparticles show a potential for 
their use as a smart material in soft sensors and actuators their application in bioengineering and medicine is doubtful due to the toxicity of metallic iron and nickel. To overcome this shortcoming the surface of metallic particles should be covered with a biocompatible layer, which prevents the contact of an open metallic surface with biological liquids. It our earlier report [24] it was shown that the surface of nickel nanoparticles produced via EEW can be modified by the in-situ carbon deposition. Such a deposition is provided by adding volatile hydrocarbons to the working gas of EEW unit. In the process of the electrical explosion hydrocarbon molecules decom- pose to elements and carbon deposits on the surface of condensing metal nanoparticles.

The modification of the surface of nickel nanoparticles opens a question on how would it influence the properties of ferrogels with embedded metal nanoparticles. In the present study we aimed to clarify two aspects of this possible influence. First, we will focus on the interfacial interaction between polyacrylamide and modified nickel nanoparticles. Second, we will address the influence of the carbon deposition on the volume swelling of ferrogels, which is a basic property for their performance in sensors and actuators.

\section{Experimental Part}

2.1. Synthesis of MNPs, composites, and ferrogels

Nickel magnetic nanoparticles (Ni MNPs) were synthesized by the electrical explosion of wire (EEW). The essence of EEW is the evaporation of a metal wire by a high-voltage electrical discharge in an inert atmosphere of argon and the subsequent condensation of metal vapors into spherical nanoparticles. Nickel nanoparticles coated with a carbon shell (Ni@C MNPs) were synthesized using a mixture of argon with the addition of butane as a working gas of EEW unit. Carbon content in the Ni@C was 2\% (wt.). The thickness of the carbon shell was 4-6 $\mathrm{nm}$. The carbon was in an amorphous state. The details of the synthetic procedure can be found in our previous reports [25].

The MNPs composites for microcalorimetry studies were prepared in the entire range of weight fraction of MNPs. Linear polyacrylamide (PAAm) was synthesized by free radical polymerization in 1.6 M water solution. Hydrogen peroxide in $18 \mathrm{mM}$ concentration was an ini- tiator of the reaction. Polymerization was done at two steps: first at $60^{\circ} \mathrm{C}$ for $30 \mathrm{~min}$, second at $100{ }^{\circ} \mathrm{C}$ for $60 \mathrm{~min}$. Molecular weight of linear PAAm was $7.3 \cdot 10^{5}$ as determined by viscometry of water solution at $25^{\circ} \mathrm{C}$. The stock solution of PAAm was then vigorously stirred with weighted amounts of Ni and Ni@C MNPs in proportions to get resulted composites with certain MNPs/PAAm ratio. The suspension of MNPs in PAAm solution was homogenized by ultra-sound treatment and then cast upon Teflon plate and dried to the constant weight at $70{ }^{\circ} \mathrm{C}$. The obtained films of NP/PAAm composites were then used for the microcalorimetry measurements of the enthalpy of dissolution in distilled water.

Ferrogels were synthesized by radical polymerization of acrylamide (AA) (Panreac Quimica SA) of in 1.6 M water solution. Methylene diacrylamide (MDAA) (MERCK) was a cross-linker in a molar ratio to monomer of 1:100. Ammonium persulfate (APS) was used as the initiator of the polymerization. Magnetic Ni/Ni@C 
nanoparticles were added to the reaction mixture in portions of a weighed $20 \%$ water-based suspension stabilized with Dispex A40 dispersant (R. T. Vanderbilt). The suspension was homogenized in an ultrasonic bath for 20 minutes. Polymerization was carried out for 60 minutes at $80{ }^{\circ} \mathrm{C}$. Synthesized ferrogels were washed in distilled water for two weeks with daily water renewal to remove residual impurities. The equilibrium swelling ratio (maximum water uptake) was determined as the ratio of the water content in the gel to the weight of the dry gel by weighing the swollen sample of the gel and the dry residue after drying to a constant weight at $70{ }^{\circ} \mathrm{C}$.

\subsection{Methods}

Transmission electron microscopy (TEM) was performed using a JEOL JEM2100 microscope operated at $200 \mathrm{kV}$. The specific surface area of MNPs was measured via low temperature adsorption of nitrogen (Brunauer-Emmett-Teller approach) using a Micromeritics TriStar3000 analyzer. Phase composition of MNPs was examined using an X-ray diffractometer Bruker D8 Discover operated at $\mathrm{Cu} \mathrm{K \alpha}$ radiation (wavelength $\lambda=1.5418 \mathrm{~A}$ ) with a graphite monochromator and a scintillation detector. XRD results were pro-

\section{Results and discussion}

\subsection{Characterization of Ni and Ni@C MNPs}

Fig. 1 shows the transmission electron microscopy (TEM) images of $\mathrm{Ni}$ and $\mathrm{Ni@C}$ MNPs. Both batches of MNPs contain individual spherical particles. A typical diameter of spherical Ni MNPs (Fig. 1a) lay within 10-100 $\mathrm{nm}$ range. Deposited thin carbon layers are clearly observed on the surface of Ni@C nanoparticles (Fig. $1 b)$. cessed using the built-in Bruker software TOPAS-3 provided the Rietveld full-profile refinement. Magnetic hysteresis loops were measured using a vibrating sample magnetometer (Cryogenics). Calorimetric measurements were done at $25^{\circ} \mathrm{C}$ using a Calvet differential microcalorimeter of laboratory design. The sensitivity was $31.5 \mathrm{mV} / \mathrm{W}$, cell volume was $10 \mathrm{~cm}^{3}$. The stability of a baseline was $\pm 0.5 \mu \mathrm{V}$. Enthalpies of dissolution of PAAm/Ni, PAAm/Ni@C, and PAAm/C composites in water were measured using glass ampoule cells. Water was placed in a stainless steel cell and small portion of a composite film (approximately $10-30 \mathrm{mg}$ by weight) was put into a thin-walled glass ampoule. This specimen was dried to a constant weight in vacuum and then the ampoule was sealed. A sealed ampoule was placed in the cell with water in it. After thermal equilibration until the baseline of calorimeter kept at a constant level the ampoule was broken inside the cell and the heat effect of the dissolution of a composite in water was measured. The absolute values of measured heat effects of dissolution were from 0.5 to 2 Joule depended on the composition of a composite. The absolute error of calorimetric measurements was 0.05 Joule.

The average diameter of MNPs was estimated based on the value of the specific surface area of MNPs, which was determined by low-temperature nitrogen adsorption. The specific surface area of $\mathrm{Ni}$ and $\mathrm{Ni@C} \mathrm{MNPs} \mathrm{obtained} \mathrm{from} \mathrm{the} \mathrm{isotherms}$ of nitrogen adsorption by the BrunauerEmmett-Teller (BET) treatment were equal to 12.6 and $10.8 \mathrm{~m}^{2} / \mathrm{g}$ respectively. Straightforward geometrical consideration of the surface of a sphere related to its 


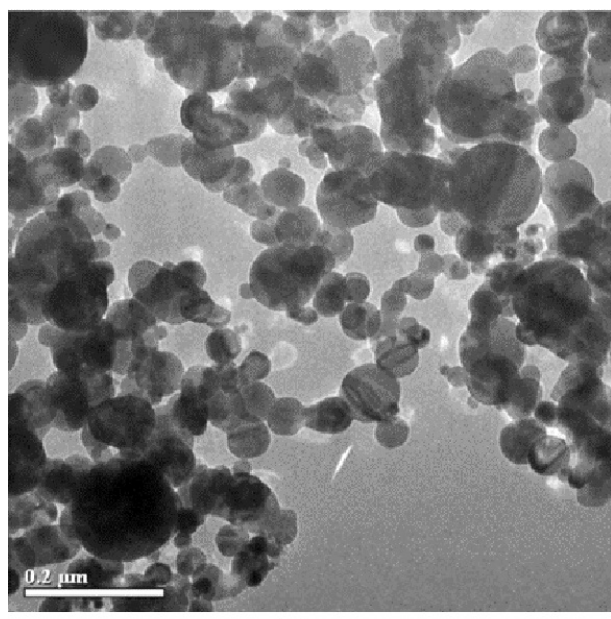

(a)

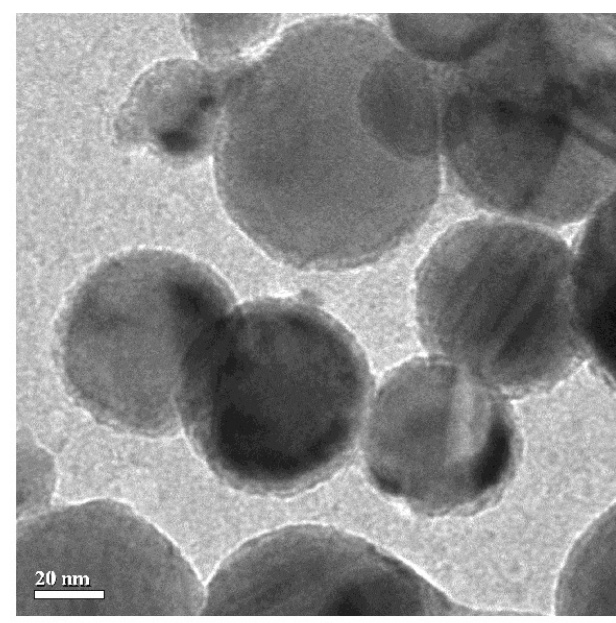

(b)

Fig. 1. TEM images of Ni (a) and Ni@C (b) MNPs.

mass gives the following simple equation for the diameter $(d)$ of the sphere in relation to its surface $(S)$ and the density $(\rho)$ of its material:

$$
d=\frac{6}{S \rho}
$$

If applied to the specific surface area of the MNPs in air-dry powder equation (1) gives the average value of the diameter for the ensemble of MNPs. If $S$ is in $\mathrm{m}^{2} / \mathrm{g}$ and $\rho$ is in $\mathrm{g} / \mathrm{cm}^{3}$ equation (1) yields the average diameter in micron. Calculated values for Ni and Ni@C MNPs are presented in Table 1. The average diameters are close to each other. The average diameter of Ni@C nanoparticles is little higher than that for Ni nanoparticles. It is consistent with the presence of a deposited carbon layer on the surface of Ni@C nanoparticles.
The phase composition of Ni and Ni@C MNPs was characterized by X-ray diffraction (XRD). According to X-ray powder diffraction data (Fig. 2), the Ni sample contained $100 \% \alpha$-Ni phase with a cubic facecentered lattice; the unit cell parameter $a=0.3523(2)$ nm. XRD pattern for Ni@C MNPs also revealed $100 \%$ a-Ni phase with

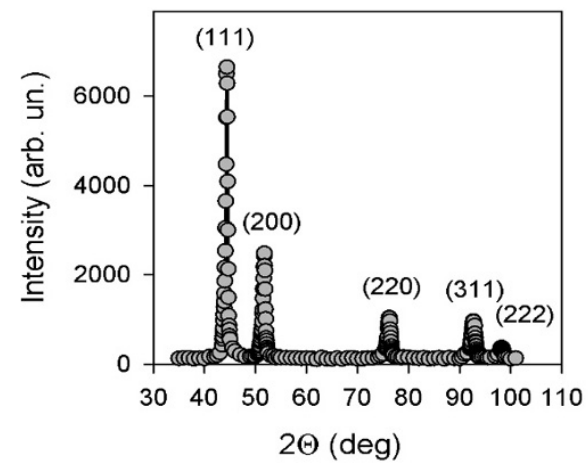

Fig. 2. XRD pattern of Ni MNPs

Table 1

Characteristics of magnetic fillers

\begin{tabular}{c|c|c|c|c|c|c}
\hline MNPs & Density, $\mathrm{g} / \mathrm{cm}^{3}$ & Shape & $\mathrm{S}_{\mathrm{sp}}, \mathrm{m}^{2} / \mathrm{g}$ & $\mathrm{d}_{\mathrm{av}}, \mathrm{nm}$ & $* \mathrm{M}_{\mathrm{s},} \mathrm{kA} / \mathrm{m}$ & $* * \mathrm{H}_{\mathrm{c}}, \mathrm{kA} / \mathrm{m}$ \\
\hline $\mathrm{Ni}$ & 8.9 & spherical & 12.6 & 58 & 454 & 20.7 \\
\hline $\mathrm{Ni} @ \mathrm{C}$ & 8.9 & spherical & 10.8 & 62 & 339 & 7.7 \\
\hline
\end{tabular}

${ }^{\star}$ - saturation magnetization; ${ }^{* *}$ - coercive force 
the unit cell parameter $a=0.3533(3) \mathrm{nm}$. Carbon layer was not detected in XRD pattern for Ni@C because the deposited layer was too thin and its content (2\%) was below the sensitivity of XRD.

Fig. 3. Shows magnetic hysteresis loops for Ni and Ni@C MNPs. In both cases magnetization reached saturation in high field range. The parameters of magnetic hysteresis loops - saturation magnetization and coercive force are given in Table 2. According to these data, Ni and Ni@C powders were soft magnetic materials with low coercive force. Saturation magnetization of Ni@C nanoparticles was by approximately $25 \%$ lower than saturation magnetization of Ni MNPs. It was likely due to the distortions of the crystalline lattice in the surface layer of Ni@C particles under the influence of deposited carbon, which could happen because of limited dissolution of carbon in the lattice.

The structural characterization of $\mathrm{Ni}$ and Ni@C MNPs showed that these nanoparticles are very much alike despite the existence of the deposited carbon layer on the surface of Ni@C MNPs. Meanwhile, this factor was of decisive importance for the interfacial properties of polymeric composites with Ni and Ni@C MNPs.

\subsection{Interfacial adhesion of PAAm to MNPs}

The basic thermodynamic property, which stands for the interaction of a polymeric chain of composite with an embedded solid particle, is the enthalpy of interfacial adhesion. The latter is the enthalpy change during the adsorption of a macromolecule on a solid surface. This process establishes adhesive contacts at the interface between a macromolecule and a particle due to molecular interactions. Such enthalpy change cannot be measured di-

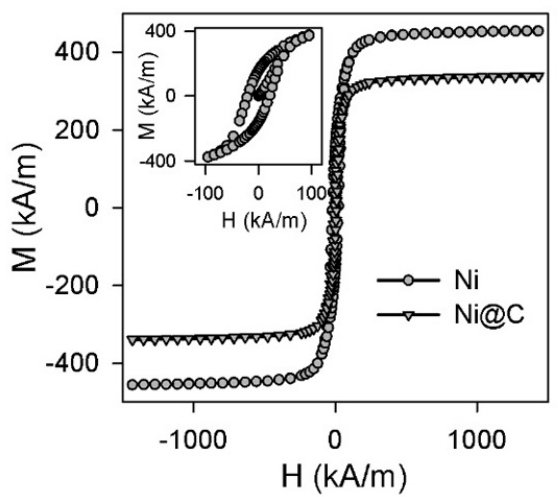

Fig. 3. Magnetic hysteresis loops for $\mathrm{Ni}$ and Ni@C MNPs. Inset - hysteresis loop in low field range for Ni MNPs

rectly in calorimetric experiment as $\mathrm{Ni}$,

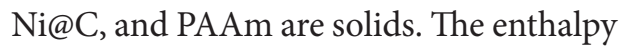
of interfacial adhesion was determined using an appropriate thermochemical cycle (Hess cycle), which included quantities measurable in calorimeter. In case of polymeric composites with embedded solid particles the enthalpy of interfacial adhesion is equal to the enthalpy of composite mixing. Since the solid particles have not dissolved in a polymeric matrix, the only source of enthalpy change is the interface interaction. The Hess cycle for the enthalpy of mixing of PAAm/Ni (or PAAm/Ni@C) composite constitutes the combination of the following processes.

1) $\mathrm{PAAm}+\mathrm{Ni}$ MNPs = Composite $\mathrm{PAAm} / \mathrm{Ni}+\Delta H_{a d h}$

2) PAAm + water (excess) = PAAm solution $+\Delta H_{d i s, p}$

3) $\mathrm{Ni}$ MNPs + water (excess) $=\mathrm{Ni}$ MNPs suspension $+\Delta H_{\text {wet }}$

4) PAAm solution + Ni MNPs suspension $=$ Ni MNPs suspension in PAAm solution $+\Delta H_{\text {mix }}$

5) Composite PAAm/Ni + water (excess $)=$ Ni MNPs suspension in PAAm solution $+\Delta H_{d i s, c}$ 
The combination of these steps gives for the enthalpy of adhesion:

$$
\begin{aligned}
\Delta H_{\text {adh }}= & \omega_{\text {PAAm }} \Delta H_{\text {dis }, p}+\omega_{N i} \Delta H_{\text {wet }}+ \\
& +\Delta H_{\text {mix }}-\Delta H_{\text {dis }, c}
\end{aligned}
$$

In Equation (2) $\omega_{P A A m}$ and $\omega_{N i}$ are weight fractions of PAAm and $\mathrm{Ni}$ in a composite; $\Delta H_{d i s, p}$ is the enthalpy of dissolution of PAAm in water; $\Delta H_{\text {wet }}$ is the enthalpy of wetting of air-dry Ni MNPs in water; $\Delta H_{m i x}$ is the enthalpy of mixing of PAAm water solution with Ni MNPs water suspension; $\Delta H_{d i s, c}$ is the enthalpy of dissolution in water for a composite with $\omega_{\text {PAAm }}$ and $\omega_{N i}$ composition.

Fig. 4 (a) shows dependencies of the enthalpy of dissolution in water versus the weight content of embedded particles for PAAm composites. Together with PAAm/Ni and PAAm/Ni@C composites we also took as a reference PAAm/Carbon composite which was prepared using commercial sample of carbon black with specific surface area $124 \mathrm{~m}^{2} / \mathrm{g}$. The value at the left-hand vertical axis corresponds to the enthalpy of dissolution of PAAm in water, which is $\Delta H_{d i s, p}$. The value at the right-hand axis corre- sponds to the enthalpy of wetting of airdry particles, which is $\Delta H_{\text {wet }}$. The symbols at the field of the plot corresponds to $\Delta H_{d i s, c}$ values for the composites with certain fractions of PAAm and a filler.

One can notice that the dependence of $\Delta H_{\text {dis, }}$ for PAAm/Ni composites is convex upward, while the dependencies of $\Delta H_{d i s, c}$ for PAAm/Ni@C and PAAm/ Carbon are concave downwards. The data presented in Fig. 4 (a) were used to calculate the enthalpy of interfacial adhesion in composites $\Delta H_{\text {adh }}$ using equation (2). It is worth noting that the values of $\Delta H_{\text {mix }}$ (step (4) of the Hess cycle) typically can be neglected as they lay within the experimental error of the other quantities of Hess cycle.

The dependence of the enthalpy of interfacial adhesion versus the content of solid particles in PAAm composites is shown in Fig. 4 (b). There is a substantial difference between dependencies of the enthalpy of adhesion for PAAm/Ni and PAAm/ $\mathrm{Ni@C} \mathrm{composites.} \mathrm{In} \mathrm{case} \mathrm{of} \mathrm{PAAm/Ni}$ composite the enthalpy of adhesion is negative over the entire composition range. It

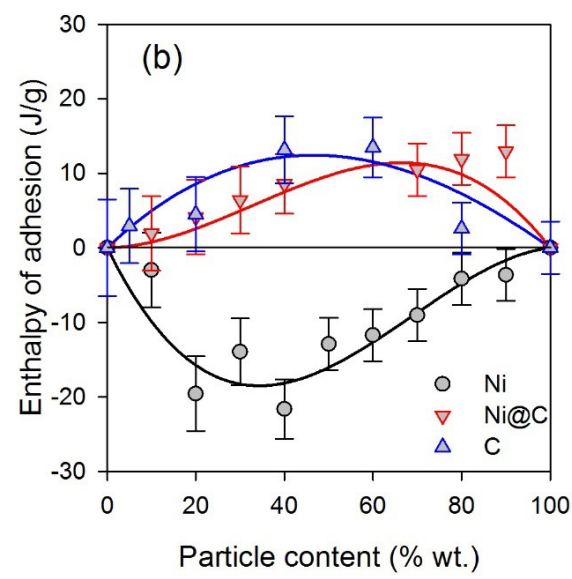

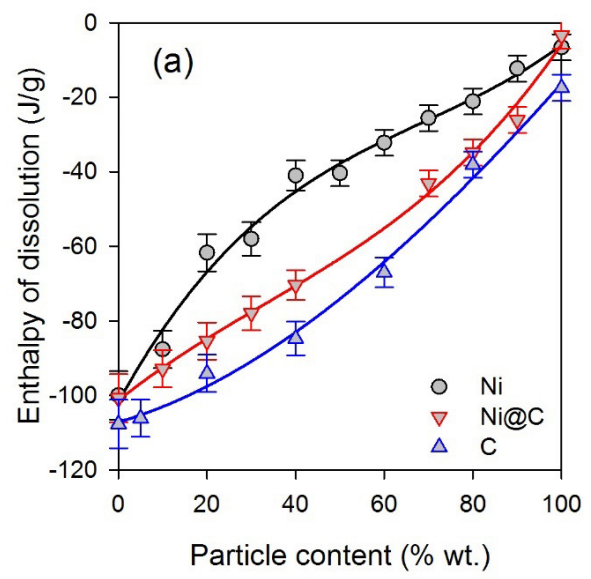

Fig. 4. a) - Concentration dependence of the enthalpy of dissolution of PAAm composites with embedded Ni, Ni@C, and C particles; b) - Concentration dependence of the enthalpy of interfacial adhesion in PAAm composites with embedded Ni, Ni@C, and C particles 
means that the adhesion contact between PAAm chain and the surface of Ni MNP is energetically favorable. Such interaction promotes adsorption of PAAm chains on the surface of Ni MNPs.

On the contrary, in case of PAAm/ $\mathrm{Ni@C} \mathrm{composite} \mathrm{the} \mathrm{enthalpy} \mathrm{of} \mathrm{adhe-}$ sion is positive over the entire composition range. It means that PAAm chains do not interact with the surface of Ni@C MNPs. No doubts that such interaction is energetically unfavorable the deposited carbon layer on the surface of MNPs. The results for the reference system PAAm/Carbon clearly confirm this fact. In the PAAm/Carbon composites the enthalpy of adhesion is also positive over the entire composition range similar to the case of PAAm $/ \mathrm{Ni@C}$ composites.

Thus, the obtained thermochemical data showed that the deposition of carbon on the surface of Ni MNPs worsened the adhesion of PAAm chains to modified Ni MNPs.

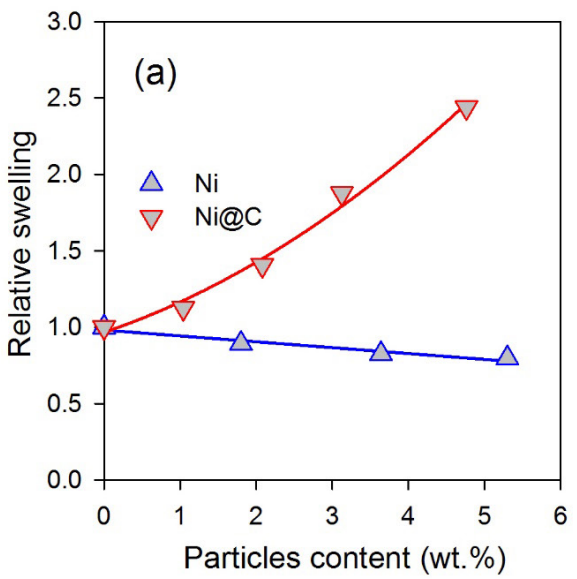

3.3. Swelling of PAAm ferrogels with $\mathrm{Ni}$ and Ni@C

Fig. 5 (a) shows the dependence of the relative degree of swelling of PAAm/ $\mathrm{Ni}$ and PAAm/Ni@C ferrogels versus the weight fraction of MNPs in ferrogel. The relative degree of swelling is the swelling ratio (water uptake) of a ferrogel divided by the swelling ratio of a blank PAAm gel with the same composition. The data presented in Fig. 7 (a) refer to gels with a network density of 100 monomer units in linear sub-chains per one cross-link. It is worth noting that the relative swelling decreases with MNPs content in the case of ferrogels with Ni MNPs, and it increases in the case of ferrogels with Ni@C MNPs.

The same trend is shown in Fig. 5 (b), which presents the swelling ratio of ferrogels with different network density containing the $3.1 \%$ (wt.) of MNPs. Both in the case of ferrogels with Ni MNPs and in the case of ferrogels with Ni@C MNPs the swelling ratio increases if the network density of a gel decreases. It is a general trend in gels. If a number of monomer

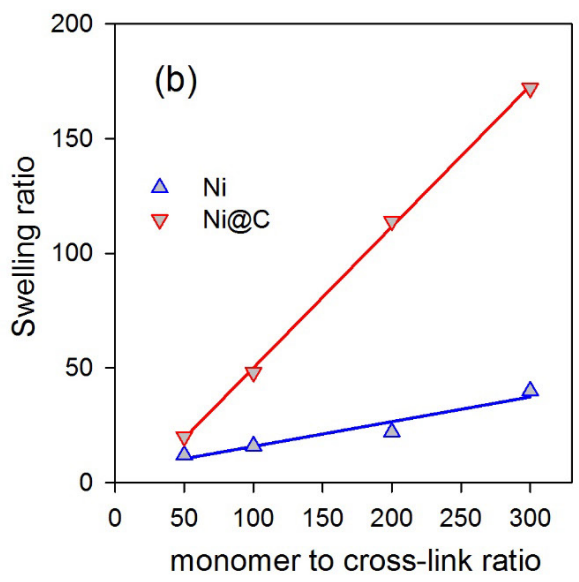

Fig. 5. a) - Dependence of the relative degree of swelling of PAAm ferrogels with Ni and Ni@C MNPs with respect to the unfilled gel matrix (monomer to cross-link ratio 100:1); b) Dependence of the swelling ratio of PAAm ferrogels with Ni and Ni@C MNPs on the length of the linear sub-chains between the crosslinks (MNPs content 3.1\%) 
units in linear sub-chains enlarge and the number of cross-links diminishes, then the network loosens and it can absorb more water. Thus the water uptake of a gel increases. Although the basic trend in the dependence of the swelling ratio on the network density is the same for both types of ferrogels, there is s remarkable difference in the absolute values of the swelling ratio. It is much higher in the case of Ni@C ferrogels. In ferrogels with the lowest network density (300 monomer units per one crosslink) the swelling ratio of PAAm ferrogel with embedded 3\% Ni@C MNPs is four times larger than that for the ferrogel with the same composition but with embedded Ni MNPs.

The reason for such a difference in swelling of ferrogels with embedded Ni or Ni@C MNPs apparently stems from the different adhesion of PAAm chains to the surface of particles. PAAm ferrogels differ from PAAm composites, because ferrogels contain water besides polymer and MNPs. Nevertheless, interaction between PAAm chains and the surface of MNPs governs the swelling of ferrogel. If interaction between polymeric chains and MNPs is strong, then the PAAm sub-chains in the network are adsorbing on the surface of the particles. Adsorption of chains effectively increases the networking density and diminishes the water uptake of the gel matrix. It is the case of PAAm ferrogel with enbedded Ni MNPs.

In turn, if PAAm chains do not interact with the surface of MNPs, then the MNPs will effectively repel the sub-chains of the networks. It will increase the mobility of the network, and it will promote extra swelling of ferrogel. It is the case of PAAm ferrogel with embedded Ni@C MNPs.

\section{Conclusions}

Metallic magnetic nanoparticles (MNPs) are prospective for the use in smart soft materials suitable for bioengineering and medical applications. However, to provide biocompatibility they need to be coated with protective layers. Deposited carbon coating is among them. Meanwhile, deposition of carbon affects functional properties of MNPs and materials, in which they are embedded. In the present study the interfacial interaction of carbon coated Ni MNPs (Ni@C) with polyacrylamide (PAAm) has been studied in comparison with Ni MNPs focusing on application in PAAm ferrogels. The interfacial adhesion of PAAm chains to the surface of MNPs was studied by means of thermochemical cycle based on calorimetric measurements of the enthalpy of dissolution of PAAm/Ni and PAAm/Ni@C composites in water, and the enthalpy of adhesion to the surface of MNps was determined in the entire range of MNPs content in the composite. It turned out that while interaction of Ni MNPs with PAAm was energetically favorable and led to the evolution of heat (negative enthalpy change) during adhesion, deposited carbon layer provided poor interaction of PAAm with the surface of Ni@C MNPs. The enthalpy of interaction between PAAm and Ni@C MNPs was positive over the entire range of MNPs content. It means that PAAm do not interact with the surface coated with carbon layer. It provided a marked impact on the swelling ratio (water uptake) of PAAm ferrogels with embedded Ni@C MNPs in comparison with ferrogels with embedded Ni MNPs. The swelling ratio of PAAm gel matrix diminished with the embedding of Ni MNPs due to the adsorption of PAAm sub-chains on the sur- 
face of MNPs. On the contrary, the embedding of Ni@C MNPs in PAAm gel matrix resulted in the increase of its swelling ratio owing to the effective repelling of PAAm chains by the carbon layer on the surface of MNPs. It means that poor interaction at the interface not necessarily worsens the functional properties of soft biocompatible materials. The increase of the swell- ing ratio might be an advantageous feature in certain applications of ferrogels in biocompatible sensors and actuators. In a whole, obtained results showed that coating of the surface of MNPs not only provides biocompatibility of MNPs but might as well be a versatile tool to modify the functional properties of smart materials based on nanoparticles.

\section{Acknowledgements}

The study was financially supported by RFBR, project number 19-38-90229.

\section{References}

1. Abasi S, Podstawczyk DA, Sherback AF, Guiseppi-Elie A. Biotechnical properties of poly(HEMA- co-HPMA) hydrogels are governed by distribution among water states. ACS Biomater. Sci. Eng. 2019;5(10):4994-5004.

DOI: 10.1021/acsbiomaterials.9b00705

2. Chen Z, Zhao D, Liu B, Nian G, Li X, Yin J, Qu S, Yang W. 3D printing of multifunctional hydrogels. Adv. Funct. Mater. 2019;29(20)1-8. doi:10.1002/adfm.201900971

3. Zhang YS, Khademhosseini A. Advances in engineering hydrogels. Science. 2017;356(6337):eaaf3627.

DOI: $10.1126 /$ science.aaf3627

4. Lui YS., Sow WT., Tan LP., Wu Y., Lai Y., Li H. 4D printing and stimuli-responsive materials in biomedical aspects. Acta Biomater. 2019;92:19-36.

DOI: 10.1016/j.actbio.2019.05.005

5. Zhou Y, Sharma N, Deshmukh P, Lakhman RK, Jain M, Kasi RM. Hierarchically structured free-standing hydrogels with liquid crystalline domains and magnetic nanoparticles as dual physical cross-linkers. J. Am. Chem. Soc. 2012;134(3):1630-1641. https://doi:10.1021/ja208349x

6. Xie W, Gao Q, Guo Z, Wang D, Gao F, Wang X, Wei Y, Zhao L. Injectable and self-healing thermosensitive magnetic hydrogel for asynchronous control release of doxorubicin and docetaxel to treat triple-negative breast cancer. ACS Appl. Mater. Interfaces 2017;9(39):33660-33673.

DOI: $10.1021 /$ acsami.7b10699

7. Noh M, Choi YH, An YH, Tahk D, Cho S, Yoon JW, Jeon NL, Park TH, Kim J, Hwang NS. Magnetic nanoparticle-embedded hydrogel sheet with a groove pattern for wound healing application. ACS Biomater. Sci. Eng. 2019;5(8):3909-3921. doi:10.1021/acsbiomaterials.8b01307

8. Tang J, Yin Q, Qiao Y, Wang T. Shape morphing of hydrogels in alternating magnetic field, ACS Appl. Mater. Interfaces. 2019);11(23):21194-21200. doi. org/10.1021/ acsami.9b05742 
9. Huang J, Liang Y, Huang Z, Zhao P, Liang Q, Liu Y, Duan L, Liu W, Zhu F, Bian L, Xia J, Xiong J, Wang D. Magnetic enhancement of chondrogenic differentiation of mesenchymal stem cells, ACS Biomater. Sci. Eng. 2019;5(5):2200-2207.

DOI: 10.1021/acsbiomaterials.9b00025

10. Huang J, Liang Y, Jia Z, Chen J, Duan L, Liu W, Zhu F, Liang Q, Zhu W, You W, Xiong J, Wang D. Development of magnetic nanocomposite hydrogel with potential cartilage tissue engineering. ACS Omega. 2018;3(6):6182-6189.

DOI: 10.1021/acsomega.8b00291

11. Zheng X, Wu D, Su T, Bao S, Liao C, Wang Q. Magnetic nanocomposite hydrogel prepared by $\mathrm{ZnO}$-initiated photopolymerization for La (III) adsorption. ACS Appl. Mater. Interfaces. 2014;6(22):19840-19849.

DOI: $10.1021 / \mathrm{am} 505177 \mathrm{c}$

12. Tang SCN, Yan DYS, Lo IMC. Sustainable wastewater treatment using microsized magnetic hydrogel with magnetic separation technology. Ind. Eng. Chem. Res. 2014;53(40):15718-15724.

DOI: $10.1021 / \mathrm{ie} 502512 \mathrm{~h}$

13. Czichy C, Spangenberg J, Günther S, Gelinsky M, Odenbach S. Determination of the Young's modulus for alginate-based hydrogel with magnetite-particles depending on storage conditions and particle concentration. J. Magn. Magn. Mater. 2020;501:166395.

DOI: $10.1016 /$ j.jmmm.2020.166395

14. Xulu PM, Filipcsei G, Zrínyi M. Preparation and Responsive Properties of Magnetically Soft Poly(N-isopropylacrylamide) Gels. Macromolecules. 2000;33(5):1716-1719. DOI: $10.1021 / \mathrm{ma} 990967 \mathrm{r}$

15. Filipcsei G, Csetneki I, Szilágyi A, Zrínyi M. Magnetic Field-Responsive Smart Polymer Composites. Advances in Polymer Science. 2007;206:137-189.

DOI: 10.1007/12_2006_104

16. Filipcsei G, Zrínyi M. Magnetodeformation Effects and the Swelling of Ferrogels in a Uniform Magnetic Field. J. Phys. Condens. 2010;22:276001.

DOI: $10.1088 / 0953-8984 / 22 / 27 / 276001$

17. Galicia JA, Sandre O, Cousin F, Guemghar D, Menager C, Cabuil V. Designing magnetic composite materials using aqueous magnetic fluids. Journal of Physics: Condensed Matter. 2003;15(15):S1379 - S1402.

DOI: $10.1088 / 0953-8984 / 15 / 15 / 306$

18. Galicia J A, Cousin F, Dubois E, Sandre O, Cabiul V and Perzynski R. Static and dynamic structural probing of swollen polyacrylamide ferrogels. Soft Matter. 2009;5(13):2614-2624.

DOI: $10.1039 / \mathrm{b} 819189 \mathrm{a}$

19. Galicia J A, Cousin F, Dubois E, Sandre O, Cabuil V and Perzynski R. Local structure of polymeric ferrogels. Journal of Magnetism and Magnetic Materials. 2011;323(10):1211-1215.

DOI: $10.1016 /$ j.jmmm.2010.11.008 
20. Shankar A, Safronov AP, Mikhnevich EA, Beketov IV. Multidomain iron nanoparticles for the preparation of polyacrylamide ferrogels. Journal of Magnetism and Magnetic Materials. 2017:431:134-137.

DOI: $10.1016 /$ j.jmmm.2016.08.075

21. Shankar A, Safronov AP, Mikhnevich EA, Beketov IV, Kurlyandskaya GV. Ferrogels based on entrapped metallic iron nanoparticles in a polyacrylamide network: extended Derjaguin-Landau-Verwey-Overbeek consideration, interfacial interactions and magnetodeformation. Soft Matter. 2017;13(18):3359-3372.

DOI: 10.1039/C7SM00534B

22. Mikhnevich EA, Chebotkova PD, Safronov AP, Kurlyandskaya GV. Influence of uniform magnetic field on elastic modulus in polyacrylamide ferrogels with embedded nickel nanoparticles. Journal of Physics: Conference Series. 2019;1389(1):012059. DOI: 10.1088/1742-6596/1389/1/012059

23. Kurlyandskaya GV, Safronov AP, Bhagat SM, Lofland SE, Beketov IV, Prieto ML. Tailoring functional properties of Ni nanoparticles-acrylic copolymer composites with different concentrations of magnetic filler. J. Appl. Phys. 2015;117:123917. DOI: $10.1063 / 1.4916700$

24. Beketov IV, Safronov AP, Medvedev AI, Murzakaev AM, Timoshenkova OR, Demina TM. In-situ formation of carbon shells on the surface of Ni nanoparticles synthesized by the electric explosion of wire. Nanosystems: physics, chemistry, mathematics. 2018;9(4):1-9.

DOI: $10.17586 / 2220-8054-2018-9-4-513-520$

25. Beketov IV, Safronov AP, Bagazeev AV, Larrañaga A, Kurlyandskaya GV, Medvedev AI. In situ modification of Fe and Ni magnetic nanopowders produced by the electrical explosion of wire. Journal of Alloys and Compounds. 2014;586:S483 - S488. DOI: $10.1016 /$ j.jallcom.2013.01.152 